\title{
Pulsar Flux Monitoring and Refractive Scintillation
}

\author{
Daniel R. Stinebring, Tatiana V. Smirnova, Jennifer Hovis, Joshua C. \\ Kempner, Edward B. Myers \\ Department of Physics, Oberlin College, Oberlin, OH 44074, USA \\ Timothy H. Hankins \\ New Mexico Technical Institute \\ Victoria M. Kaspi \\ Jet Propulsion Laboratory $\&$ California Institute of Technology \\ David J. Nice \\ National Radio Astronomy Observatory
}

\begin{abstract}
We have monitored the $610 \mathrm{MHz}$ flux density of 21 pulsars on a daily basis for five years. The flux density time series for these pulsars range from nearly constant for the most distant and heavily scattered pulsars to rapidly varying, saturated time series for more nearby pulsars. The measured stability of the flux density from the most distant pulsars (variations less than $5 \%$ ) implies that the average radio emission from pulsars, before it has been affected by propagation through the ISM, is constant in strength over five years. The flux variations for 12 of the pulsars are consistent with a Kolmogorov turbulence spectrum over a range of more than three orders of magnitude in scattering strength, with no detectable presence of an inner scale $\left(s_{i} \geq 10^{7} \mathrm{~cm}\right)$. The flux variations are greater than predicted by this model for five pulsars - including the Crab and Vela - but this group is consistent with a Kolmogorov spectrum and an inner scale of $\sim 10^{10} \mathrm{~cm}$.
\end{abstract}

\section{Introduction}

The interstellar medium is inhomogeneous on a wide range of length scales, from the size of discrete structures in spiral arms $(100 \mathrm{pc})$ down to $10^{8} \mathrm{~cm}$ or smaller. Radio waves from pulsars propagating through this medium are slowed by interaction with free electrons, giving rise, for example, to the dispersion of the signal. Inhomogeneities in the medium cause scattering of the radio waves, which is responsible for a variety of related phenomena: angular broadening of pulsars, temporal smearing of pulsed signals, and multi-path interference which, in turn, gives rise to scintillation (flux variation) of the signal in both time and frequency. We monitored the flux of 21 pulsars at a frequency of $610 \mathrm{MHz}$ on a 
nearly daily basis from May, 1990, to May, 1995, using a 26-m diameter telescope in Green Bank, West Virginia. The goal of this project was to improve our understanding of the interstellar medium on length scales in the range $10^{12} \mathrm{~cm}$ to $10^{14} \mathrm{~cm}$. The flux variations we measure allow us to probe the spectrum and the distribution of inhomogeneities in the interstellar medium. Rickett (1990) provides a good review of refractive and diffractive scintillation.

\section{Observations}

The observations reported here were made at a frequency of $610 \mathrm{MHz}$ with a $2 \times 16 \times 1 \mathrm{MHz}$ filter bank and the Pulsar Mark 3 data acquisition system. A typical hardware integration cycle ( $a$ 'scan') for each pulsar is between 2 and 10 minutes depending on the signal strength. Each pulsar was observed for between 10 minutes and 2 hours per day to yield a reasonable signal to noise ratio and to smooth the diffractive scintillations. For the 1847 day duration of this project we obtained usable flux values for about $55 \%$ of the days for most of the pulsars.

The first scan for each pulsar observation is a noise diode calibration scan. These calibration scans are of fundamental importance to this project since they form our primary power reference level. The set of flux estimates for the day's observation are put through a procedure to eliminate estimates which have too low a signal-to-noise ratio, are corrupted by interference, or are highly discrepant.

In all cases, the nearly constant flux time series are for pulsars that are distant, heavily scattered, have low transverse velocity, or a combination of all three. A quantitative analysis of this leads us to the conclusion that pulsars are constant flux radio sources over time scales of hours to five years.

\section{Data Analysis}

From a structure function analysis of the data we can determine a) the saturation value, b) the refractive time scale, and c) the logarithmic slope or slopes for lags much shorter than the saturation time scale.

The logarithmic slope of the intensity structure function, $\gamma$ is a valuable indicator of the distribution of scattering material along the line of sight and the predominant value of the inhomogeneity spectral index, $\beta$. The value of $\gamma$ can distinguish clearly between a model in which the scattering material is predominantly located in a thin screen along the line of sight $(\gamma \approx 2)$ and the case in which the main scattering material is distributed more uniformly along the line of sight $(\gamma=\beta-3)$.

\section{Results}

We present a summary of our results in Table I, where $T_{\text {ref,obs }}$ is the observed refractive time scale (time lag at one-half the saturation level) and the modulation index is the ratio of the r.m.s. flux variation to the mean flux level.

A quantitative analysis of the time series and the corresponding structure functions for these 21 pulsars leads to the following conclusions: 


\begin{tabular}{|c|c|c|c||c|c|c|c|}
\hline PSR & DM & $\begin{array}{c}T_{\text {ref,obs }} \\
\text { [day] }\end{array}$ & $\begin{array}{c}\text { mod. } \\
\text { index }\end{array}$ & PSR & DM & $\begin{array}{c}\text { Tref,obs } \\
\text { [day] }\end{array}$ & $\begin{array}{c}\text { mod. } \\
\text { index }\end{array}$ \\
\hline $0136+57$ & 73.7 & 25 & 0.15 & $1642-03$ & 35.7 & 0.9 & 0.46 \\
$0329+54$ & 26.8 & 15 & 0.37 & $1749-28$ & 50.9 & 28 & 0.25 \\
$0525+21$ & 50.9 & 4.0 & 0.31 & $1818-04$ & 84.4 & - & 0.10 \\
$0531+21$ & 56.8 & 12 & 0.32 & $1831-03$ & 235.8 & - & 0.07 \\
$0736-40$ & 160.8 & 71 & 0.03 & $1859+03$ & 402.9 & - & 0.03 \\
$0740-28$ & 73.8 & 29 & 0.13 & $1911-04$ & 89.4 & 24 & 0.21 \\
$0818-13$ & 41.0 & 12 & 0.23 & $1933+16$ & 158.5 & 33 & 0.24 \\
$0833-45$ & 69.1 & 35 & 0.24 & $1946+35$ & 129.0 & - & 0.05 \\
$0835-41$ & 147.6 & 10 & 0.21 & $2111+46$ & 141.5 & 72 & 0.15 \\
$1556-44$ & 58.8 & 15 & 0.27 & $2217+47$ & 43.5 & 5.4 & 0.30 \\
$1641-45$ & 480.0 & - & 0.05 & & & & \\
\hline
\end{tabular}

Table 1. Observed refractive scintillation parameters

- Distant pulsars have nearly constant flux $(\delta F / F \leq 5 \%)$.

- No pulsar in our sample has a modulation index that exceeds 0.5.

- There is reasonable agreement between observed and predicted refractive time scales, but the observed values are low by a factor of $2-4$ for the more distant pulsars.

- The modulation index of most of the pulsars in our sample are consistent with a Kolmogorov inhomogeneity spectrum with an inner scale no larger than $\approx 10^{7} \mathrm{~cm}$.

- These pulsars constrain the exponent of the inhomogeneity spectrum to lie in the range $3.6 \leq \beta \leq 3.75$.

- Several pulsars, located within supernova remnants or behind young HII regions, have a modulation index consistent with a Kolmogorov inner scale model (or, similarly a Shishov "two-scale" model) with an inner scale of around $10^{10} \mathrm{~cm}$.

- The logarithmic slope of the structure function generally lies in the range $0.6<\gamma<1.2$, indicating that the scattering material is either uniformly distributed along the line of sight or at least is not absent from the vicinity of the pulsar.

- We find numerous examples of structure functions with a break in the logarithmic slope indicating a steepening in the inhomogeneity spectrum in accord with the model of Shishov (1993).

\section{Discussion}

Several of these results appeared in an analysis of the first 400 days of this monitoring program (Kaspi \& Stinebring 1992). With a significantly longer time 
span, however, we are able to study the structure functions of the flux time series in a definitive manner. We confirm our earlier result that pulsar fluxes appear to be intrinsically stable over several years. We find that many of the pulsars in our sample, for which we now have well determined modulation indices, are consistent with a Kolmogorov turbulence model for ISM inhomogeneities with a small $\left(\approx 10^{7} \mathrm{~cm}\right)$ inner scale.

One of the most striking results to come from this analysis is a group of pulsars (PSRs 0531+21, 0833-45, 0835-41, 1911-04, and 1933+16) which display a modulation depth significantly larger than the Kolmogorov value. These could be explained by an inner scale of around $10^{10} \mathrm{~cm}$. A preliminary examination of the lines of sight which show greater than expected modulation shows that four of the five (PSR 1911-04 is the apparent exception) intersect young supernova remnants or HII regions. We note that most of the pulsars which show anomalously large modulation also display a break in the logarithmic structure function slope.

A more complete report of these observations and analysis is in preparation.

\section{References}

Kaspi, V. M. \& Stinebring, D. R. 1992, ApJ, 392, 530

Rickett, B. J. 1990, ARA\&A, 28, 561

Shishov, V. I. 1993, Soviet Ast., 37, 378 